\title{
Beobachtungen zur Stabilimentvariabilität bei adulten Weibchen der Wespenspinne (Argiope bruennichi) im Kreis Siegen-Wittgenstein (NRW)
}

\author{
Uwe DIENER
}

Abstract: Stabilimentum variability in the webs of adult female Argiope bruennichi

Bei einer Bestandserhebung im Zusammenhang mit der weiteren Ausbreitung der mediterran-subatlantischen Wespenspinne in NordrheinWestfalen konnten im Kreis Siegen-Wittgenstein (DIENER in Vorber.) die folgenden Beobachtungen gemacht werden.

1998 wurde zwischen Geisweid und Birlenbach MTB 5013/4.4 eine Population mit 73 Weibchen gefunden. Die Netzanlagen $(n=77)$ wurden nach Netzfläche und Höhe über dem Boden vermessen. Charakteristisch für ein Wespenspinnennetz sind die beiden senkrechten Stabilimente oberund unterhalb der Nabe. Bei vier Netzen $(5,2 \%)$ waren diese Stabilimente nicht senkrecht, sondern als Zickzackband rund, wobei der Kreis unten immer ca. $5 \mathrm{~mm}$ offen war. Die Funddaten der Netze mitrunden Stabilimenten waren der 17./ 18. und 28. August. Die Größen der Netzflächen lagen im Bereich der Durchschnittsgröße der vermessenen Netze.

KREUELS (briefl.) sind Netzänderungen aus chemisch kontaminierten Gegenden bekannt. Diese Ursache trifft hier nicht zu, da die Wiesen zum Teil nicht genutzt werden und zum anderen der Viehhaltung dienen und nicht mit Spritzmitteln behandelt werden. Behandelte Äcker sind in der Nähe ebenfalls nicht vorhanden.

SACHER (1991) berichtet in seiner Arbeit über rudimentäre Radnetze bei adulten Männchen der Wespenspinne, daß die Männchen bis zum Erreichen der Geschlechtsreife Radnetze mit Stabilimenten weben, die entweder nach oben und unten verlaufen oder als dichte, circuläre Struktur aus bis zu 6 Bändern bestehen. Abb. 4 in seiner Arbeit zeigt ein unreifes Weibchen mit einer Kombination aus circulären Strukturen und dem von der Nabe aus nach oben und unten verlaufendem Zickzackband im Netz. Desweiteren gibter an, daß noch adulte Männchen Radnetze weben, deren 
Stabilimente von der Nabe aus nach oben und unten verlaufen oder nur 1 Band nach unten haben. Eine dritte Variante ist das Fehlen des Stabiliments. MALT (briefl.) ist eine kreisförmige Stabilimentstruktur bei Weibchen nur in juvenilen Entwicklungsstadien im Zeitraum Ende Mai / Juni bekannt. Subadulte und adulte Radnetze der Weibchen in der von inm beobachteten Population im Leutratal bei Jena wiesen zweiarmige Stabilimente auf.

Als Grund der beschriebenen runden Stabilimente adulter Weibchen vermuten KORDGES (mündl.) und KREUELS (briefl.) eine Störung des Verhaltensmusters. MALT (briefl.), der sich eingehend mit der Wespenspinne befaßt hat, interpretiert solche Anlagen als eine wahrscheinlich alterungsbedingte Änderung des Verhaltensmusters beim Netzbau während des letzten Lebensabschnitts von Argiope-Weibchen.

Bei den 1999 kontrollierten Netzen $(n=12)$ konnten keine runden Stabilimente gefunden werden. Lediglich zweiNetze hatten am 26.September das Stabiliment nur unterhalb der Nabe. MALT (briefl.) fand solche Netze ebenfalls in der von inm beobachteten Population und führt an, daß gegen Ende der Reifeperiode etwa ab Ende August die Zahl der zweiarmigen Stabilimente stark abnahm.

Dank: Herrn Dr. Steffen MALT, Jena gilt mein besonderer Dank für seine ausführlichen Anmerkungen zur Problematik der Stabilimentvariabilität und der kritischen Beurteilung der Beobachtungen. Herrn Dr. Martin KREUELS, Münster danke ich für den konstruktiven Briefwechsel mit vielen Anmerkungen und Anregungen.

\section{LITERATUR}

SACHER, P. (1991): Rudimentäre Radnetze bei adulten Männchen der Wespenspinne Argiope bruennichi. - Veröff. Naturhist. Mus. Schleusingen 6: 30-38

Uwe DIENER, Am Grünen Hang 10, D-57078 Siegen 